Derecho \& Realidad

Vol. 13 - Núm. 25 •Enero-Junio de 2015

Págs. 93-110 • ISSN:1692-3936

\title{
Una justificación de los derechos humanos sociales*
}

\author{
A justification of the social human rights
}

\author{
Hugo Ómar Seleme** \\ hugoseleme@conicet.gov.ar
}

\section{Resumen}

Más allá del debate de derecho positivo referido a si los derechos sociales han recibido en la normativa internacional el estatus de genuinos derechos, existe un debate filosófico-político que se pregunta por su justificación moral. ¿Existen derechos humanos de índole moral-que pueden coincidir o no con los reconocidos por el derecho positivo- cuyo contenido sean exigencias sociales o económicas? Aunque las preguntas por el estatus moral y el estatus jurídico son diferentes, lo que se responda en el primer caso puede tener incidencia sobre cómo pensamos que debería diseñarse el sistema jurídico internacional. Argumentar a favor de la existencia de derechos morales humanos con contenido social puede ser una razón-aunque no una definitivapara justificar su positivización.

Fecha de aceptación: 18 de septiembre de 2014

Fecha de recepción: 15 de octubre de 2014

Fecha de aprobación final: 20 de febrero de 2015

* Artículo de reflexión, producto en la línea de investigación proyecto de investigación ha sido "legitimidad, justicia y globalización"

* Catedrático de Ética, Facultad de Derecho, Universidad Nacional de Córdoba (Argentina). Profesor del Heidelberg Center for Latin America.Investigadordel CONICET. Director de la Maestría en Derecho y Argumentación. 
Este artículo se ocupa solamente del problema filosófico-político, dejando de lado el debate centrado en el derecho positivo. El objetivo es ofrecer los principales lineamientos de una justificación moral de los derechos humanos. Dicha justificación permite no solo dar cuenta de algunos rasgos peculiares de los derechos humanos, sino que posibilita comprender que las mismas razones morales que subyacen a los derechos civiles y políticos se aplican a los derechos sociales. El argumento posee dos partes. La primera tiene por objetivo elaborar la justificación propuesta. Una vez expuesta la justificación de los derechos humanos, la segunda parte del argumento tiene por objetivo mostrar que la misma sirve para dar sustento no solo a los derechos humanos civiles y politicos, sino también a los derechos humanos sociales.

\title{
Palabrac clave
}

Derechos humanos sociales, justificación moral de los derechos humanos.

\begin{abstract}
Beyond the debate on positive law referred to whether social rights have received international status of genuine rights, exists a philosophically debate that asks about their moral justification. Are there any human rights of moral nature that may coincide or not with the recognized by positive law-whose contents are social or economic demands? Although the questions about the moral status and legal status are different, which is answered in the first case may have impact on how we think the international legal system should be designed. Arguing for the existence of moral rights with social content may be one reason -although not a definitive- to justify their fixing in positive rules.

This article addresses only the problem philosophically, leaving aside the debate centered on the positive law. The objective is to offer the main guidelines of a moral justification of human rights. Such a justification not only allows giving information of some peculiar features of human rights, but it makes it possible to understand that the same moral reasons that underlie the civil and political rights apply to social rights. The matter has two parts. The first aims to develop the proposed justification. Once exposed the justification of human rights, the second part aims to show that it serves to sustain not only civil and political rights, but also social human rights.
\end{abstract}

\section{Keywords}

Social human rights, moral justification of human rights. 


\section{Introducción}

Los derechos humanos se han transformado en el lenguaje del discurso político internacional. Las estructuras institucionales domésticas son alabadas o criticadas utilizándolos como métrica. Las políticas de los Estados son evaluadas teniendo en mente el grado de satisfacción de este tipo específico de estándar normativo. Acusar a un Estado de vulnerar los derechos humanos de sus ciudadanos es una de las imputaciones más graves que pueden formulársele.

La importancia que poseen los derechos humanos en el discurso internacional y la gravedad que implica su vulneración por parte de un Estado, queda patente en la institución de la intervención humanitaria. La violación masiva de los derechos humanos por parte de un Estado justifica la intervención de otro Estado -aun la armada- con el objetivo de poner fin a tal trasgresión ${ }^{1}$. Aunque existe disputa en el ámbito internacional acerca de cual es la justificación jurídica de este tipo de intervención humanitaria -debido a que la Carta de las Naciones Unidas solo contempla la intervención que tiene por objeto poner fin a potenciales amenazas para la paz (art.7)- tanto a nivel filosófico ${ }^{2}$ como a nivel político ${ }^{3}$ su aceptación es amplia.

La importancia de los derechos humanos en el discurso político internacional hizo que desde la aprobación misma de la Declaración de los Derechos Humanos comenzase un movimiento para que los derechos sociales -algunos de los cuales se encontraban enunciados en la Declaración ${ }^{4}-$ fuesen incluidos en un tratado que les concediese estatus de norma de derecho internacional. Esta tarea de intentar concertar tratados sobre los derechos consignados en la Declaración -entre ellos los derechos sociales- fue confiada a la Comisión de Derechos Humanos. Debido a las tensiones y divisiones generadas por la guerra fría, la tarea de acordar sobre un tratado de

\footnotetext{
El concepto restringido de intervención humanitaria solo las circunscribe al caso de intervenciones armadas. Una utilización del mismo puede encontrarse en Vincent (1974, p. 8). El concepto amplio, por el contrario, considera cualquier injerencia en los asuntos internos de otro Estadocon el objeto de proteger los derechos humanos como intervención humanitaria. Medidas económicas tales como el embargo o el otorgamiento de incentivos serían tipos de intervención humanitaria. Una utilización del concepto amplio de intervención humanitaria puede encontrarse en Beitz (19791999, p. 73-75).

2 David Luban fue uno de los primeros en defender la tesis de que la intervención en un país extranjero con el objeto de defender los derechos humanos se encontraba justificada, aunque violase la soberanía y pudiese calificarse según el orden legal internacional como una agresión (1980, p. 160-181).

3 La resolución 60/1 de la Asamblea General de las Naciones Unidas, dictada el 24 de octubre del 2005, por la cual aprobó el informe de la International Comission on Intervention and State Sovereignty,es una prueba contundente de que existe aceptación política en el ámbito internacional con respecto a la intervención humanitaria.

4 La Declaración contemplaba el derecho al trabajo, la alimentación y a la educación.
} 
derechos sociales se volvió impracticable, por lo que la Comisión optó por trabajar en dos tratados separados, uno referido a derechos civiles y políticos y otro a derechos económicos, sociales y culturales. Ambos tratados fueron presentados para su aprobación a la Asamblea General en 1953, lo que finalmente sucedió recién en $1966^{5}$.

La aprobación y ratificación del Pacto de Derechos Sociales, Económicos y Culturales, sin embargo, no logró zanjar la cuestión de si estos derechos -al igual que los civiles y políticos- eran genuinos derechos humanos. El problema radica en el tipo de garantía diferente que se requiere por parte del Estado en relación con ambos conjuntos de derechos. El Pacto de Derechos Sociales, Económicos y Culturales establece exigencias más débiles sobre los Estados que las estipuladas por el Pacto de Derechos Civiles y Políticos. Aquel solamente exige que los Estados adopten medidas para lograr progresivamente la plena efectividad de los derechos sociales, económicos y culturales ${ }^{6}$, lo que contrasta con lo establecido con respecto a los derechos civiles y políticos, donde simplemente se exige que cada Estado respete y garantice los derechos civiles a todos los individuos que se encuentran en su territorio y están sometidos a su jurisdicción ${ }^{7}$. Los derechos sociales parecen ser reconocidos más como directrices políticas que como genuinos derechos.

Los tratados recibieron el número de ratificaciones necesarias para volverse operativos recién en 1976.

6 El Pacto de Derechos Sociales, Económicos y Culturales establece en su artículo 2: 1. Cada uno de los Estados Partes en el presente Pacto se compromete a adoptar medidas, tanto por separado como mediante la asistencia y la cooperación internacionales, especialmente económicas y técnicas, hasta el máximo de los recursos de que disponga, para lograr progresivamente, por todos los medios apropiados, inclusive en particular la adopción de medidas legislativas, la plena efectividad de los derechos aquí reconocidos. 2. Los Estados Partes en el presente Pacto se comprometen a garantizar el ejercicio de los derechos que en él se enuncian, sin discriminación alguna por motivos de raza, color, sexo, idioma, religión, opinión política o de otra índole, origen nacional o social, posición económica, nacimiento o cualquier otra condición social. 3. Los países en desarrollo, teniendo debidamente en cuenta los derechos humanos y su economía nacional, podrán determinar en qué medida garantizarán los derechos económicos reconocidos en el presente Pacto a personas que no sean nacionales suyos. (ONU, 2012)

7 Por su parte, el Pacto de Derechos Civiles y Políticos señala en su artículo 2: 1. Cada uno de los Estados Partes en el presente Pacto se compromete a respetar y a garantizar a todos los individuos que se encuentren en su territorio y estén sujetos a su jurisdicción los derechos reconocidos en el presente Pacto, sin distinción alguna de raza, color, sexo, idioma, religión, opinión política o de otra índole, origen nacional o social, posición económica, nacimiento o cualquier otra condición social. 2. Cada Estado Parte se compromete a adoptar, con arreglo a sus procedimientos constitucionales y a las disposiciones del presente Pacto, las medidas oportunas para dictar las disposiciones legislativas o de otro carácter que fueren necesarias para hacer efectivos los derechos reconocidos en el presente Pacto y que no estuviesen ya garantizados por disposiciones legislativas o de otro carácter. 3. Cada uno de los Estados Partes en el presente Pacto se compromete a garantizar que: a) Toda persona cuyos derechos o libertades reconocidos en el presente Pacto hayan sido violados podrá interponer un recurso efectivo, aun cuando tal violación hubiera sido cometida por personas que actuaban en ejercicio de sus funciones oficiales; b) La autoridad competente, judicial, administrativa o legislativa, o cualquiera otra autoridad competente prevista por el sistema legal del Estado, decidirá sobre los derechos de toda persona que interponga tal recurso, y desarrollará las posibilidades de recurso judicial; c) Las autoridades competentes cumplirán toda decisión en que se haya estimado procedente el recurso. (ONU, 2012) 
Más allá del debate de derecho positivo referido a si los derechos sociales han recibido en la normativa internacional el estatus de genuinos derechos, existe un debate filosófico-político que se pregunta por su justificación moral. ¿Existen derechos humanos de índole moral -que pueden coincidir o no con los reconocidos por el derecho positivo- cuyo contenido sean exigencias sociales o económicas? Aunque las preguntas por el estatus moral y el estatus jurídico son diferentes, lo que se responda en el primer caso puede tener incidencia sobre cómo pensamos que debería diseñarse el sistema jurídico internacional. Argumentar a favor de la existencia de derechos morales humanos con contenido social puede ser una razón -aunque no una definitiva- para justificar su positivización.

En lo que sigue pretendo ocuparme solamente del problema filosófico-político, dejando de lado el debate centrado en el derecho positivo. Mi objetivo es ofrecer los principales lineamientos de una justificación moral de los derechos humanos. Dicha justificación permite no solo dar cuenta de algunos rasgos peculiares de los derechos humanos, sino que posibilita comprender que las mismas razones morales que subyacen a los derechos civiles y políticos se aplican a los derechos sociales. El argumento posee dos partes. La primera tiene por objetivo elaborar la justificación propuesta. Una vez expuesta la justificación de los derechos humanos, la segunda parte del argumento tiene por objetivo mostrar que la misma sirve para dar sustento no solo a los derechos humanos civiles y políticos, sino también a los derechos humanos sociales.

\section{Coacción y derechos humanos}

La idea central de la justificación de los derechos humanos que ofreceré es la siguiente. Los derechos humanos son las exigencias morales que aparecen como consecuencia de dos rasgos del orden internacional: su carácter coercitivo y su carácter estatal. El orden internacional impone de modo coercitivo sobre todos los individuos que habitan el planeta un sistema de Estados, los que a su vez poseen instituciones que se aplican de modo coercitivo. Que los derechos humanos sean la contrapartida del carácter coercitivo del orden internacional, y que existan dos niveles de coacción -el internacional y el estatal- es lo que explica uno de los rasgos centrales de los derechos humanos, a saber, que los Estados sean los principales responsables de su satisfacción y que la comunidad internacional aparezca como garante. El recurso extremo que posee la comunidad internacional para efectivizar esta garantía es el de la intervención humanitaria.

El sentido en que considero que las instituciones domésticas e internacionales son coercitivas se aparta de la idea tradicional de coerción. Según la idea tradicional, una regla o conjunto de reglas tiene carácter coercitivo cuando su cumplimiento se encuentra respaldado por la amenaza de una sanción o por el uso de la fuerza. 
La coerción es entendida aquí como interferencia ${ }^{8}$. Si no existe coerción, esto es, si no hay interferencia, entonces el sujeto es libre, en el sentido de que puede conducirse como le place sin soportar obstrucciones o amenazas por parte de otros (Pettit \& Lovett, 14). El mal de la coerción, entonces, reside en que un esquema institucional respaldado por amenazas hace que los sujetos a quienes se aplica no tengan el control sobre sus propias existencias, al privarles del dominio sobre sus decisiones. Si existe coerción, el control está en poder de otro. La amenaza de castigo incide sobre las opciones disponibles, haciendo que la probabilidad de que elijan el curso de acción penado disminuya -siempre que el individuo no quiera afrontar mayores costos- de modo proporcional a la gravedad del castigo ponderada por la probabilidad de que se haga efectivo. En este sentido, el individuo no es el autor -no tiene el control- de su decisión.

No obstante, si uno toma como relevante para la existencia de coacción el hecho de que otro tenga control sobre las decisiones de un individuo, no existe razón alguna para entender que las instituciones coercitivas son solo aquellas respaldadas por el uso efectivo o la amenaza del uso de la fuerza. La existencia de un esquema institucional vuelve más probable que un individuo opte por cierto curso de acción o soporte un determinado estado de cosas, con total independencia de que el mismo imponga sanciones a la no realización de la acción en cuestión o utilice la fuerza o la amenaza del uso de la fuerza para que determinado estado de cosas se materialice. El modo en que un esquema institucional

recompensa los talentos naturales, o la manera en que distribuye el ingreso o las oportunidades, determinan que sea más probable para un individuo, por ejemplo, optar por una carrera en lugar de otra -optar por cierto curso de acción- o no disponer de ciertos recursos o bienes -soportar un estado de cosas-.

Para que exista coacción, en el sentido que lo estoy utilizando, tienen que configurarse dos elementos. En primer lugar, el esquema institucional tiene que aumentar la probabilidad de que un curso de acción sea adoptado o un estado de cosas sea soportado por los sujetos a quienes se aplica. En segundo lugar, dicho efecto tiene que ser producido intencionalmente. No basta, en consecuencia, que un esquema institucional tenga por efecto condicionar las alternativas de las que disponen los sujetos a quienes se aplica o producir un determinado estado de cosas, sino que adicionalmente es necesario que esté dirigido a producir este resultado.

\footnotetext{
Arthur Ripstein señala como representante paradigmático de la concepción tradicional a Stuart Mill. La coerción: “....interferes with a person's liberty, and does so by imposing a cost on that person that he or she would not otherwise have borne..." (Ripstein, 2004, p. 2-35). Philip Pettit, por su parte, traza el origen de la noción de libertad como no-interferencia que subyace a la concepción tradicional de coerción hasta Hobbes (Pettit, 2008; Pettit \& Lovett, 2009, p. 15).
} 
La afrenta de la coerción no reside simplemente en que la voluntad de un agente sea anulada -no tenga control- sino en que su voluntad sea reemplazada por otra, una intención ajena tiene control sobre él. Para que un esquema institucional sea coercitivo, es necesario que persiga el efecto de aumentar la probabilidad de un curso de acción o el acaecimiento de un estado de cosas ${ }^{9}$.

Para determinar los agentes que son coercionados por un esquema institucional es necesario prestar atención a quiénes el esquema institucional pretende imponer un estado de cosas o curso de acción. Así, si un esquema institucional incide sobre la probabilidad que un agente elija un curso de acción o soporte un estado de cosas pero no era esa la pretensión del esquema, dicho agente no es sujeto de coerción. Esto nos deja con el problema de determinar sobre quiénes un esquema institucional pretende incidir, el cual no es más que una instancia del problema más general referido a cómo determinar sobre quiénes recaen las consecuencias intencionales de un esquema institucional.

Thomas Pogge (1990, p. 45) ha trazado una distinción entre las consecuencias producidas intencionalmente por las instituciones y aquellas que no lo $\operatorname{son}^{10}$. Lo primero que Pogge distingue son las consecuencias que los esquemas institucionales producen -aquellos efectos que no se producirían en ausencia del esquema institucional-, de aquellas que meramente deja acaecer-aquellos efectos que acaecen con independencia de la existencia del esquema institucional-. Las consecuencias producidas intencionalmente -aquellas que serían consideradas relevantes por un enfoque deontológico- son un subconjunto de las consecuencias producidas. Específicamente, se trata de las consecuencias establecidas de modo directo por el esquema institucional a través de sus reglas. Por ejemplo, una consecuencia establecida por las reglas que crean una economía de mercado es que las personas puedan comprar y vender. Una consecuencia producida, pero no establecida sino meramente engendrada, del mismo conjunto de reglas es que algunas personas que no tienen nada de valor para ofrecer caigan por debajo de la pobreza. Esta última

\footnotetext{
Con ligeras modificaciones he estado interpretando coerción del mismo modo que Pettit utiliza el concepto de dominación. No obstante, aqu me aparto de su posición, lo que justifica no utilizar la misma terminología. En efecto, para Pettit puede existir dominación sin la intención de dominar. Pettit señala: "One person, A, controls the choice of another person, B, when A does something that has the intentional or quasi-intentional effect of raising the probability that $B$ will choose according to A's taste or judgment -raising it beyond the probability that this would have had in A's absence..." (Pettit, 2009, p. 42). Pienso que esto trae aparejado consecuencias indeseables. Una de ellas es que todo acto que produce externalidades que inciden sobre la probabilidad que otro adopte un curso de acción debe ser considerado un acto de dominación. Introducir el requisito de la intencionalidad tiene la ventaja de permitir trazar la distinción entre un acto de coerción -o dominación intencional- y uno que produce meras externalidades.

10 Sigo aquí lo que he señalado, con ligeras modificaciones, en otros trabajos (Seleme, 2007, p. 1-60; y 2009, p. 205-228)
} 
consecuencia es producida -ya que estas personas presumiblemente no serían pobres si existiese otro conjunto de reglas que regulase la economía- pero no es establecida en tanto no hay ninguna regla que fije de modo explícito su nivel de ingresos.

Tomando la distinción de Pogge, puede señalarse, entonces, que un esquema institucional pretende incidir solamente sobre aquellos a quienes establece como destinatarios, no sobre todos en quienes produce consecuencias. Las consecuencias que un esquema institucional previsiblemente engendra, sirven para determinar a qué han sido coaccionados aquellos a quienes establece como destinatarios. Así, por ejemplo, en el caso del esquema institucional que organiza la economía de mercado dentro de un Estado, las personas a quienes se aplica coercitivamente serán quienes habitan el territorio del mismo, a quienes las normas establecen como destinatarios. La consecuencia engendrada de la pobreza servirá para determinar a qué han sido coercionados algunos de estos ciudadanos. Si la pobreza, como una consecuencia engendrada, recae tanto sobre sujetos a quienes el esquema establece como destinatarios como sobre quienes no lo son, dado el carácter intencional de la coacción, solo de los primeros puede afirmarse que de modo coactivo han sido puestos en una situación de pobreza. Los segundos son solo víctimas de una externalidad negativa producida por el esquema institucional, pero no son sujetos a quienes el esquema institucional se aplica coactivamente. Así, si las instituciones que organizan la economía de mercado en un Estado tienen efectos colaterales en quienes habitan un Estado vecino -por ejemplo, alterando su nivel de ingreso debido al aumento o disminución del comercio con el país vecino- los ciudadanos del segundo Estado no están siendo coercionados por el esquema institucional del país vecino. Están sufriendo o disfrutando de sus consecuencias, pero en tanto aquel esquema no los establece como destinatarios, falta el requisito de la intencionalidad y, por tanto, no hay coacción ${ }^{11}$.

Tanto las instituciones domésticas como las internacionales poseen carácter coercitivo en el sentido señalado. En ambos casos, su existencia aumenta la probabilidad de que los sujetos a quienes establecen como destinatarios adopten cierto curso de acción o soporten algún estado de cosas. En lo que se distinguen es en el tipo de sujeto a quienes establecen como destinatarios -esto es, a quienes coercionan- y el

$\overline{11}$ En mi opinión esta es la noción rawlsiana de instituciones coercitivas. Que una institución se aplique coercitivamente no se encuentra vinculado al uso o a la amenazade uso de la fuerza. No obstante soy consciente de que esta interpretación no es pacífica. Así, por ejemplo, Michael Blake presupone en su argumento que Rawls se encuentra comprometido con la idea tradicional de coacción (Blake, 2002, p. 257-296). Gerald Cohen señala dos modos alternativos de interpretar en qué consisten las instituciones básicas según Rawls, uno de los cuales es semejante a la idea de instituciones de aplicación coercitiva que presento aquí. Cohen sostiene que sea que uno opte por una noción restringida de estructura básica -coercitiva en el sentido tradicional-o una más amplia -coercitiva en el sentido que utilizo en el texto- la concepción rawlsiana se encuentra en problemas (Cohen, 2000, p. 134-148). 
tipo de curso de acción o estado de cosas cuya probabilidad aumentan -esto es, a qué son coercionados-.

El sujeto paradigmático a quienes se aplican las instituciones domésticas estatales son las personas consideradas aisladamente, los individuos que habitan un determinado territorio. El esquema institucional que los establece como destinatarios aumenta la probabilidad de que cada ciudadano goce de ciertas expectativas vitales, derechos y oportunidades. Si el esquema institucional que establece a ciertos individuos como destinatarios contiene reglas que organizan el libre mercado, reconocen el derecho a la herencia, reconocen y protegen a la familia, y no fijan mínimos vitales, es probable que los individuos que nacieron en familias con altos recursos gocen de mayores oportunidades y expectativas vitales que aquellos que nacieron en una familia pobre. Más aun, es probable que los individuos nacidos en familias pobres y que no poseen ninguno de los talentos naturales premiados por el mercado, no tengan ni siquiera los mínimos recursos para subsistir. De modo coercitivo, el esquema institucional fija las expectativas tanto de quienes favorece como de quienes perjudica. Aumenta la probabilidad de que acaezcan ciertos estados de cosas. Lo mismo puede decirse respecto del aumento de probabilidades de que algunos individuos adopten un curso de acción. En un esquema institucional como el presentado, es más probable que los individuos nacidos en familias con bajos recursos, menores oportunidades y expectativas vitales, opten por delinquir ${ }^{12}$.

Las instituciones internacionales se diferencian de las domésticas en dos sentidos. En primer lugar, establecen como destinatarios tanto a los individuos considerados aisladamente como a los individuos organizados políticamente, esto es, a los Estados. Mientras las instituciones domésticas se aplican a individuos, las internacionales adicionalmente lo hacen a los Estados. En segundo lugar, aun cuando se aplican a individuos el curso de acción o estado de cosa cuya probabilidad aumenta tiene características distintivas que lo diferencian de los efectos que producen las instituciones domésticas. ${ }^{13}$

La característica más relevante de las instituciones internacionales es que establecen un sistema de unidades políticas territoriales -los Estados- que poseen autoridad dentro de sus fronteras. A su vez, las reglas institucionales del esquema internacional

12 Sobre la correlación entre pobreza y delincuencia existen múltiples trabajos. Por todos, puede verse el de Andrew Karmen. En el mismo se muestra a partir de datos de la ciudad de Nueva York que las personas que habitan una situación de pobreza no solo tienen más probabilidades de delinquir -lo que muestra que las instituciones aumentan la probabilidad de que opten porun curso de acción- sino también de ser víctimas de delitos -lo que muestra que las mismas instituciones aumentan la probabilidad de que les acaezca un estado de cosas- (Karmen, 2000, p. 26-34).

13 Una peculiaridad adicional del orden internacional radica en que los encargados de aplicar este orden institucional internacional son los mismos Estados, bien individualmente o de modo colectivo. 
establecen como destinatarios a los Estados. Estas, por ejemplo, fijan sus derechos y deberes en relación con otros Estados, tal como el derecho a disponer de los recursos naturales que se encuentran en su territorio, el deber de no intervenir en los asuntos de otros Estados, el deber de no llevar adelante guerras de agresión o el de respetar los tratados. Estas reglas poseen carácter coercitivo en tanto aumentan la probabilidad de que ciertos estados de cosas acaezcan a los Estados -por ejemplo, que unos dispongan de mayores recursos naturales que otros- o adopten ciertos cursos de acción -por ejemplo, que un Estado respete las decisiones que sobre cuestiones domésticas adopta otro-.

Ahora bien, el hecho de que las instituciones internacionales establezcan un sistema de Estados, determina que también sean coercitivas con relación a los individuos, no en tanto miembros de una organización política de base territorial, sino en tanto tales. El orden internacional de modo coercitivo establece un sistema estatal, esto es, un sistema cuyas unidades -los Estados- son a su vez esquemas institucionales que se aplican coercitivamente a los individuos. El hecho de que no quede ningún territorio libre del control estatal a donde un individuo que no aceptase esta forma de organización política pudiese emigrar, es una de las consecuencias que el orden internacional de modo coercitivo impone sobre todos los individuos que habitan el planeta ${ }^{14}$. Cada individuo es coercionado por el esquema de instituciones internacionales a habitar un esquema de instituciones estatales que, a su vez, se le aplican coercitivamente.

En consecuencia, aunque tanto el esquema de instituciones domésticas como internacionales se aplican coercitivamente a los individuos, aquello a lo que los coercionan es diferente. El esquema internacional les coerciona a vivir en algún Estado, aunque no les coerciona a vivir en el Estado particular en que lo hacen. Aumenta la probabilidad de que un estado de cosas acaezca -el de que habiten algún esquema estatal- e incide sobre la probabilidad de que adopten un curso de acción -el que no puedan optar por vivir en un territorio sin Estado-. El esquema de instituciones domésticas, en cambio, los coerciona a vivir en el particular conjunto de instituciones domésticas que los establecen como destinatarios. Estas, como

14 En este punto discrepo con Pettit, quien señala que este hecho no puede considerarse que sea uno impuesto intencionalmente o cuasi-intencionalmente por un agente. Pettit señala que "...It is a brute fact or historical necessity -an obstacle created by nature- that there is no State-less territory available..." (Pettit, 2009, p. 52, y s.f.). Pettit considera que este hecho no es "...in itself the effect of dominating interference by the local state," y de allí concluye que es un hecho bruto. Aunque considero que lo primero es cierto, pienso que la conclusión no se sigue. Esto toda vez que aún es posible, como sostengo, que se trate de un efecto producido por la "interferencia dominante" o la aplicación coercitiva de un esquema institucional distinto al estatal, esto es, el esquemade instituciones internacionales. Si existiese otro orden internacional -distinto al construido por la Paz de Westfalia- seguramente el hecho de que todo el territorio se encuentre repartido entre Estados internamente supremos y externamente independientes, no sería el caso. Este estado de cosas no es un hecho bruto, sino uno producido por ciertas instituciones. 
hemos visto, aumentan la probabilidad de que a quienes se aplican les acaezcan ciertos estados de cosas -tales como los referidos al nivel de sus expectativas vitales, recursos y derechos- o adopten ciertos cursos de acción -tales como seguir una carrera u otra, o en el caso extremo delinquir o no hacerlo-.

El esquema de instituciones internacionales no afecta a los individuos de modo directo -más allá del hecho señalado de imponerles vivir en algún Estado- fijando sus expectativas vitales, recursos y derechos. El esquema de instituciones internacionales puede aumentar la probabilidad de que los Estados se encuentren organizados de cierto modo -por ejemplo, tengan gobiernos estables y legítimos o posean dictaduras inestables- pero aun con esta probabilidad en pie, lo que aumenta la probabilidad de que los ciudadanos en particular se encuentren en cierto estado de cosas -respecto de los recursos, derechos y oportunidades que reciben- es el modo en que el Estado efectivamente se organiza.

El hecho de que un determinado esquema de instituciones estatales se aplique coercitivamente a un individuo da lugar a que se produzca un tipo de afrenta moral, lo que engendra ciertas exigencias morales que deben ser satisfechas para evitarla. La afrenta consiste en que los deberes y derechos, los cursos de acción, las oportunidades, los recursos, los fines e intereses, de los sujetos a quienes se impone el esquema coercitivo sean determinados por alguien distinto a ellos mismos. Es el caso cuando existe un esquema institucional con profundos efectos sobre los sujetos a quienes se aplica, que a su vez es ajeno a los sujetos a quienes se impone. La posible afrenta a la que da lugar la existencia de un esquema coercitivo estatal radica en la imposición de un esquema institucional ajeno. Esta es la afrenta moral que sufren, por ejemplo, los sujetos que habitan un Estado tiránico, en cuyo diseño los individuos están impedidos de participar.

Este problema que presenta la existencia de un esquema institucional coercitivo -que sea ajeno a los individuos a quienes se aplica- engendra una exigencia moral: que todos los sujetos a quienes se aplica sean sus autores. No se trata de la exigencia de que el esquema institucional sea justo o correcto, sino de que sea propio de los sujetos a quienes se aplica. La exigencia moral que engendra la aplicación de las instituciones estatales -las cuales son coercitivas y tienen profundos efectos sobre aquellos a quienes se aplican- es la de legitimidad política ${ }^{15}$.

\footnotetext{
15 Justicia y autoría -o legitimidad política- son dos cosas diferentes. Un conjunto de sujetos pueden ser autores de un esquema institucional -puede existir legitimidad política y estarse autogobernandoaunque el esquema institucional sea injusto o incorrecto. Así como una decisión individual puede ser propia y no obstante equivocada, un esquema institucionalpuede ser propio de los sujetos a quienes se aplica, ser legítimo, y no obstante ser injusto o incorrecto. Lo contrario implicaría afirmar que cuando una decisión o esquema institucional es propia de un individuo, entonces indefectiblemente es justa o correcta, lo cual no es el caso. Mis errores son míos, pero lo mismo siguen siendo errores.
} 
Ahora bien, aunque estas exigencias de legitimidad deben ser satisfechas por las instituciones estatales -o lo que es lo mismo, son exigencias cuya satisfacción se deben recíprocamente todos aquellos individuos que habitan un mismo Estado-, también cabe cierta responsabilidad al orden internacional. El Estado -en tanto orden coercitivo que tiene profunda influencia sobre las expectativas vitales de sus ciudadanos- es el primer responsable de la legitimidad de sus instituciones. El esquema de instituciones estatales es el primer responsable en tratar a sus ciudadanos como autores. No obstante, en tanto el esquema de instituciones internacionales es el que ha creado e impone de modo coercitivo el sistema de Estados, él debe satisfacer una exigencia moral de segundo orden. Debe contener garantías que permitan asegurar que todos los Estados tratan a sus ciudadanos como autores, esto es, satisfacen las exigencias de legitimidad. Las instituciones internacionales -en tanto imponen coercitivamente un sistema de Estados- deben garantizar que los esquemas institucionales estatales protejan los derechos que son condiciones de su legitimidad. Este tipo de exigencia moral que las instituciones internacionales imponen sobre las estatales y de las cuales aparecen como garantes son los derechos humanos.

El hecho de que el esquema de instituciones internacionales de modo coercitivo establezca un sistema de Estados -que a su vez son esquemas que coercitivamente se aplican a quienes los habitan- determina que el carácter coercitivo de las instituciones estatales no solo engendre exigencias morales respecto a cómo deben encontrarse diseñadas las instituciones estatales, sino también al modo en que dicho diseño debe ser garantizado por las instituciones internacionales. Las instituciones internacionales deben garantizar que las instituciones estatales -cuya existencia es coercitivamente impuesta por el orden internacional- se organicen de modo legítimo.

Lo señalado explica un rasgo peculiar de los derechos humanos, a saber, que su satisfacción es una exigencia que en primer lugar compete a cada Estado respecto a sus propios ciudadanos y, en calidad de garante, a la comunidad internacional. Los derechos humanos son requerimientos morales cuyos destinatarios son los Estados, siendo la comunidad internacional un mero garante de segundo orden. Los Estados deben satisfacer los requerimientos de legitimidad engendrados por la coacción y el esquema internacional garantiza -a través de la exigencia de los derechos humanos- que así lo hagan.

De modo que para saber cuál es el contenido de los derechos humanos, es necesario preguntarse por el contenido de las exigencias de legitimidad. Una vez determinadas las características que un esquema estatal debe poseer para ser legítimo, es posible establecer qué contenido poseen los derechos humanos, que actúan como garantes de que estas características se encuentren satisfechas. A ello dedicaré la sección siguiente. 


\section{Legitimidad estatal y derechos humanos}

Un esquema institucional es legítimo cuando aquellos a quienes se aplica son sus autores. La autoría o legitimidad no se encuentra vinculada a lo que los individuos hacen respecto al esquema institucional -participando, consintiendo, etc.- sino en lo que las instituciones hacen respecto de los sujetos a quienes se aplican. Los sujetos a quienes se aplican las instituciones no son autores del diseño institucional porque lo configuren a través de su participación efectiva, sino que es el diseño institucional el que los configura como autores. Si un esquema institucional satisface los intereses que los sujetos a quienes se aplica poseen en tanto autores, entonces los transforma en tales ${ }^{16}$.

Puesto que las instituciones domésticas estatales se aplican coercitivamente a los ciudadanos considerados individualmente, son sus intereses los moralmente relevantes. Si dichas instituciones satisfacen los intereses que los ciudadanos tienen en tanto autores, entonces son de su autoría. Puesto que el principal interés que -en tanto autores- poseen los ciudadanos en relación con sus instituciones es el de participar efectivamente en su diseño y configuración, si las instituciones posibilitan tal cosa, entonces son de su autoría, esto es, son legítimas.

La participación posee dos manifestaciones. La primera se refiere a formar parte en la toma de decisiones colectivas. La segunda se refiere a la aceptación de las decisiones colectivas adoptadas. De modo que existen dos modos en que un esquema institucional puede no tratar como autores, no satisfacer los intereses que tienen como participantes, los ciudadanos. Por impedirles que sus opiniones u intereses cuenten a la hora de tomar decisiones colectivas. Por tratarlos como meros súbditos, receptores de órdenes, de quienes no se pretende aceptación sino solo obediencia.

Siguiendo a Charles Beitz es posible identificar tres grupos de intereses vinculados con la ciudadanía: el interés en el reconocimiento, en el modo de tratamiento ${ }^{17}$ y en la responsabilidad deliberativa. En lo que sigue me detendré a mostrar qué porción de estos intereses se encuentra vinculada a los dos aspectos antes señalados de la participación, esto es, cuáles de estos intereses son intereses que los ciudadanos tienen como autores.

El interés en el reconocimiento se encuentra vinculado al acceso a los roles públicos y a la participación en los procedimientos decisorios. Este interés se refiere a los efectos que tiene sobre la identidad pública el lugar que el procedimiento político de

${ }_{16}$ El modo de vincular coacción y legitimidad, así como el modo de concebir la legitimidad ha sido expuesto en otros trabajos, a los que sigo aquí. (Seleme, 2007 y 2010)

7 Beitz lo denomina "interés en el tratamiento equitativo" (Betiz, 1990, p. 109) 
toma de decisión colectiva y la estructura de roles públicos asigna a los individuos. Se trata de intereses que los ciudadanos tienen en su calidad de autores, puesto que se encuentran vinculados a su calidad de participantes. Cuando las instituciones están diseñadas de tal modo que una persona es excluida enteramente del acceso a cualquier rol público o cuando los roles en los procedimientos decisorios reflejan la creencia social en la inferioridad de un grupo, el interés en el reconocimiento que todos los ciudadanos tienen como autores de las instituciones que se les aplican no es satisfecho. Si una persona o grupo de individuos es excluido del acceso a los roles públicos, y tal cosa se encuentra fundada en la creencia social de su inferioridad, entonces el esquema institucional no trata a los excluidos como autores, como participantes ${ }^{18}$.

El segundo de estos intereses, el referido al modo de tratamiento, se encuentra vinculado al tipo de participación que se realiza a través de la aceptación del esquema institucional por parte de los ciudadanos. El esquema debe estar diseñado de tal modo de posibilitar dicho involucramiento de la voluntad de aquellos ciudadanos a quienes se aplica. Un tipo de esquema institucional que no hace posible la aceptación por parte de sus ciudadanos, es uno que solo descansa en el uso de la fuerza. Un esquema donde una parte de la población fuese esclava y estuviese obligada a trabajar a favor de otros, o no tuviese garantizado los medios materiales de subsistencia a través de los derechos sociales, o no tuviese protección en contra del maltrato o el homicidio, o no pudiese profesar cierta religión o pensamiento, o no tuviese garantías en contra del trato arbitrario por parte de las autoridades, sería uno que solo descansaría en el uso de la fuerza. El esquema institucional no sería uno en el cual sus instituciones se mantienen en vigor por la aceptación de su población -la que de este modo es participante y autor- sino meramente por el uso de la fuerza de sus gobernantes. Sin estos derechos, la idea misma de sistema político -como sistema de cooperación social de cuyas decisiones son autores los ciudadanos- carece de sentido ${ }^{19}$.

En consecuencia, para satisfacer los intereses que los ciudadanos tienen en tanto autores, para posibilitarles participar como aceptantes, el esquema institucional tiene que garantizar ciertos derechos tanto de índole civil como social. El derecho a la vida, a los medios de subsistencia y a la integridad personal, la libertad de

18 Beitz senala que un procedimiento decisorio con estas características "...establish or reinforce the perception that some people's interests deserve less respect or concern than those of others simple in virtue of their membership in one rather than another social or ascriptive group..." (Beitz, 1990, p. 110). Para que esto se dé, sin embargo, no basta que algunas personas sean excluidas. Es necesario, además, que tal exclusión se deba a la creencia de que poseen menos valor.

19 Considero que este es el argumento que justifica los derechos humanos en la concepción política rawlsiana. Son derechos que los individuos deben poseer si van a ser considerados "autores" de las decisiones de su sistema político. Señala Rawls: "...What have come to be called human rights are recognized as necessary conditions of any system of social cooperation. When they are regularly violated, we have command by force, a slave system, and no cooperation of any kind" (Rawls, 1999, p. 68). 
ocupación forzosa y de conciencia, la igualdad de trato por las autoridades, etc. Si estos derechos no están satisfechos, los ciudadanos no son tratados como autores y el esquema institucional es ilegitimo. ${ }^{20}$ Estos derechos, y el de que ningún grupo puede ser excluido del acceso a los roles públicos en base a consideraciones fundadas en su inferioridad, son condiciones necesarias que cualquier esquema institucional debe satisfacer para ser legítimo. Si estos derechos no se encuentran satisfechos, el esquema institucional carece de legitimidad.

Finalmente, el tercer tipo de interés se refiere a semejanza del primero a la posibilidad de participar en los procedimientos de toma de decisión colectiva. Se trata del interés en que la resolución de los asuntos políticos pueda hacerse en base a una deliberación pública suficientemente informada, donde las opiniones o razones puedan ser consideradas y evaluadas responsablemente. Este tercer interés es satisfecho cuando las instituciones son sensibles a las opiniones e intereses que sobre los asuntos públicos tienen los ciudadanos. Un sistema de toma de decisiones colectivas que impide que sean consideradas las opiniones de determinado grupo no satisface este interés en la responsabilidad deliberativa.

Entonces, si un esquema institucional estatal concede a los ciudadanos los derechos y libertades políticas que les permiten acceder a los roles públicos y hacer escuchar sus opiniones -tales como el derecho político a elegir a sus representantes y a ser elegidos, a peticionar a las autoridades, a expresar sus opiniones, etc.- y les garantiza los derechos civiles y sociales que hacen posible que el esquema institucional sea aceptado y no solo obedecido, entonces el esquema institucional los trata como autores $\mathrm{y}$, por tanto, es legítimo.

Estos derechos -civiles, políticos y sociales- son las condiciones de legitimidad de todo esquema institucional estatal. Si estos derechos se encuentran protegidos por el esquema institucional estatal, los intereses de autoría de los ciudadanos se encuentran satisfechos y la exigencia moral que surge del carácter coactivo de las

20 En este punto he seguido la exposición que Rawls realiza con relación a las condiciones que un esquema institucional debe satisfacer para ser un esquema de cooperación. Rawls contrapone dicho esquema con lo que denomina una "slave society". En la terminología que he utilizado, tal sociedad sería una que no permitiría que sus ciudadanos fuesen aceptantes, esto es, descansaría meramente en el uso de la fuerza. Señala Rawls: "...A slave society lacks a decent system o flaw, asits salve economy is driven by as cheme of political and social cooperation..." (Rawls, 1999, p. 65). A diferencia de Rawls, sin embargo, no considero que los derechos humanos puedan reducirse a aquellos que garantizan que losciudadanos puedan ser aceptantes. Los derechos humanos garantizan la legitimidad de un esquema institucional -evitando que sea una "slave society"- pero para hacerlo deben ser más extensos que lo que Rawls piensa. Básicamente deben incluir los derechos que permiten satisfacer los otros dos intereses vinculados con la autoría. Artículo de reflexión, producto de la tesis doctoral del autor, titulada: Empresa y DerechosHumanos en Colombia. Confluencia entre los modelos de Desarrollo y Derechos Humanos 
instituciones estatales se encuentra saldada. No obstante, como hemos señalado, todavía restan otras exigencias surgidas del carácter coactivo no ya del orden estatal sino del internacional. Para satisfacer los intereses de autoría de los individuos sobre quienes impone un sistema de Estados, el orden internacional debe exigir y garantizar que los Estados satisfagan las condiciones de legitimidad antes señaladas. Esto es, debe exigir que los Estados protejan los derechos civiles, sociales y políticos que son condiciones suficientes de legitimidad y cuya protección implica que los intereses de autoría de los ciudadanos se encuentren satisfechos. Dicho de modo sintético, así como el hecho de que los esquemas estatales protejan los derechos civiles, sociales y políticos es condición de su legitimidad, del mismo modo que el orden internacional proteja los derechos humanos es condición de la suya.

\section{Conclusión}

Las mismas razones que justifican que el orden internacional exija de los Estados la protección de los derechos civiles y políticos, permiten extender dicha exigencia a la protección de los derechos sociales. Sobre el orden internacional recae la exigencia moral -surgida de la imposición coercitiva de un sistema internacional estatal- de garantizar que los Estados protejan los derechos civiles, sociales y políticos de sus ciudadanos. Sobre los Estados recae la exigencia moral -surgida de la imposición coercitiva de sus instituciones sobre los ciudadanos- de garantizar la protección de los derechos civiles, sociales y políticos que son condiciones suficientes de su legitimidad. Si un Estado no protege los derechos sociales de sus ciudadanos no los trata como autores y es, por tanto, ilegítimo. Si el orden internacional no exige esta protección -a través de la positivización de derechos humanos sociales- carece igualmente de legitimidad. En el primer caso, las exigencias morales surgidas de la imposición coactiva de un particular esquema de instituciones estatales quedan insatisfechas. En el segundo, las que no son satisfechas

son las exigencias morales surgidas de la imposición de un sistema internacional de Estados por parte del orden internacional.

\section{Referencias}

Beitz, C. (1979-1999). Political theory and international relations. Princeton: Princeton University Press.

Beitz, C. (1990). Political equality: an essay in democratic theory. Princeton: Princeton University Press.

Blake, M. (2002). Distributive justice, state coertion, and autonomy. Philosophy and Public Affairs 30, 257-296. 
Cohen, G. (2000). If you're an egalitarian, how come you're so rich? Cambridge, Mass: Harvard University Pres.

Karmen, A. (2000). Poverty, crime and criminal justice. In W. Heffernan \& J. Kleining (eds.). From social justice to criminal justice. Poverty and the administration of criminal law. Oxford: Oxford University Press.

Luban, D. (1980). Just war and human rights. Philosophy and Public Affairs, 9, $160-181$

ONU, (2012, mayo). Pacto de Derechos Sociales, Económicos y Culturales. Recuperado de http://www2.ohchr.org/spanish/law/cescr.htm

Pettit, P. (s.f. en prensa). Legitimate international institutions: a new-republican perspective. In S. Besson \& J. Tasioulas (eds.).The Philosophy of International Law. Oxford: Oxford University Press.

Pettit, P. (2008). Made with words: hobbes on language, mind and politics. New Jersey: Princeton University Press.

Pettit, P. (2009). Law and Liberty. In S. Besson \& J.L. Martí (eds.). Legal republicanism: national and international perspectives. Oxford: Oxford University Press.

Pettit, P. \& Lovett, F. (2009). Neorepublicanism: a normative and institutional research program. Annual Review of Political Science 12, 11-29.

Pogge, T. (1990). Realizing Rawls. Ithaca: Cornell University.

Ripstein, A. (2004). Authority and coercion. Philosophy and Public Affairs 32, 2 35 .

Seleme, H. O. (2007). Legitimidad política, justicia y globalización. Revista Argentina de Teoría Jurídica, 9, 1-60.

Seleme, H. O. (2009). La consistencia del contextualismo institucional e internacional. Enrahonar, 43, 205-228.

Seleme, H. O. (2010). Derechos sociales y legitimidad política. En S. Ribotta \& A. Rossetti (eds.). Los derechos sociales como exigencias de justicia del siglo XXI. (pp. 173-185). Madrid: Dikinson.

Rawls, J. (1999). The law of peoples. Cambridge, Massachusetts: Harvard University Press.

Vincent, J. R. (1974). Nonintervention and international order. Princeton: Princeton University Press. 